\title{
Tetrahedron classes based on edge lengths
}

\author{
Karl Wirth and André S. Dreiding
}

Karl Wirth was a mathematics teacher in Zürich. Since his retirement he is concerned with mathematical problems in connection with chemical structures.

André S. Dreiding is professor emeritus for organic chemistry at the University of Zürich. He persues his interests in mathematically oriented aspects of chemistry.

\section{Tetrahedron e-classes}

Among triangles (the simplexes in 2-space) there are 3 kinds, the equilateral, the isosceles and the scalene. Which analogous kinds of tetrahedrons (the simplexes in 3-space) can be distinguished? Obviously, a regular tetrahedron (all edge lengths equal), at one extreme, corresponds to the equilateral triangle and a completely irregular tetrahedron (all edge lengths mutually different), at the other extreme, corresponds to the scalene. However, while there is only 1 kind, the isosceles, between the 2 extreme triangle kinds, there are 23 kinds between the 2 extreme tetrahedron kinds, thus 25 in total.

How do we arrive at these 25 tetrahedron kinds? To explain, we replace the more colloquial 'tetrahedron kind' by the concept of 'tetrahedron e-class' based on vertex maps: If $T$ and

Es gibt gleichseitige, gleichschenklige und ungleichseitige Dreiecke. Wie sieht die entsprechende Klassifizierung bei Tetraedern aus? Am einen Ende der Skala befindet sich das gleichseitige Tetraeder, am andern Ende Tetraeder mit lauter unterschiedlich langen Kanten. Die Autoren der vorliegenden Arbeit finden dazwischen 23 Klassen von Tetraedern. Dabei sind nebst der Symmetriegruppe $\mathcal{S}$ auch die sogenannte Permetriegruppe $\mathcal{P}$ und die resultierende Longometriegruppe $\mathcal{L}:=\mathcal{P} / \mathcal{S}$ Klasseninvarianten. Exemplarisch wird dargelegt, wie diese Gruppen, die für beliebige Polytope definierbar sind, algorithmisch ermittelt werden können. Aus der Ordnung der Kantenlängen ergibt sich sodann eine verfeinerte Klassifzierung von Tetraedern, deren Klassen sich mit Hilfe von $\mathcal{L}$ abzählen lassen. Diese Klassen werden innerhalb von einfachen grösseren Klassen hinsichtlich Repräsentierbarkeit mit kleinsten ganzahligen Kantenlängen untersucht. Schliesslich geht es noch um die Anzahl entsprechender Simplexklassen in höheren Dimensionen. 
$T^{\prime}$ are two tetrahedrons we call a bijection $f$ which maps the vertexes of $T$ onto the vertexes of $T^{\prime}$ a vertex map from $T$ to $T^{\prime}$.

Definition 1.1. Let $T$ be a tetrahedron. A tetrahedron $T^{\prime}$ belongs to the $e$-class represented by $T$ and denoted by $[T]_{\mathrm{e}}$ if there exists a vertex map $f$ from $T$ to $T^{\prime}$, so that $f$ induces a bijection between equal edge lengths of $T$ and those of $T^{\prime}$ or, in other words, so that both edge maps induced by $f$ and by $f^{-1}$ preserve the lengths $e$ quality. We call such a vertex map $f$ an e-metry from $T$ to $T^{\prime}$.

If $f$ is an e-metry, the induced bijection of edge lengths is denoted by $\lambda(f)$. Fig. 1 shows an example of an e-metry $f$ from $T$ to $T^{\prime}$ and we have $\lambda(f): 6 \mapsto 9,8 \mapsto 5,7 \mapsto 4$.
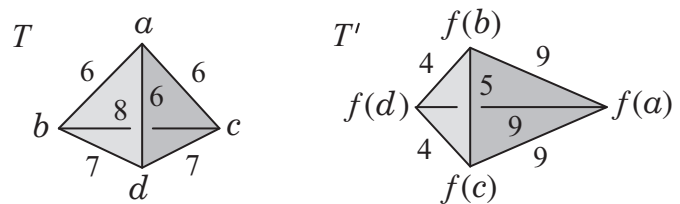

Fig. 1

In the special case of an e-metry $f$ where $\lambda(f)$ is the identity, we speak of an isometry (this uniquely determines an 'isometry' in its ordinary sense, i.e., a length preserving map of the whole space onto itself).

Of course, the e-classes form a (set) partition of all tetrahedrons. By constructive combinatorics we now generate the e-classes in showing how Tab. 1 is obtained: In the first column, the number $n$ of different edge lengths, called lengths number, varies from 1 to 6 . In the second column, the distributions of the edge lengths correspond to the 11 (number) partitions of 6 , i.e., $6,5+1, \ldots, 1+1+1+1+1+1$, for short written as $6,51, \ldots, 11111$, denoted by $m$ and named lengths partition. In each row with a given lengths partition $m$, the different arrangements of the edges are elaborated. This leads to a total of 25 cells where each cell contains a drawing of a tetrahedron (edge lengths differentiated by line formats) representing one of the 25 e-classes: $\left[T_{1}\right]_{\mathrm{e}},\left[T_{2}\right]_{\mathrm{e}}, \ldots,\left[T_{25}\right]_{\mathrm{e}}$; this classification is also found in [2]. The further information within the cells will be explained in the following Section 2.

Remark. So far, we have tacitly assumed that the tetrahedrons under consideration actually exist. But if one admits any six lengths there are those that are not the edge lengths of a tetrahedron, a circumstance which was originally treated by Menger, Blumenthal, Herzog and others and was elaborated in a survey article [5]. In this paper, given edge lengths will always define a tetrahedron.

\section{Symmetry and permetry groups}

Based on e-metries, we consider what we call permetries as a conceptual extension of the well known symmetries of a tetrahedron:

Definition 2.1. An e-metry of a tetrahedron $T$ onto itself is called a permetry. If $p$ is a permetry of $T$, the induced bijection $\lambda(p)$ is said to be a longometry of $T$. 


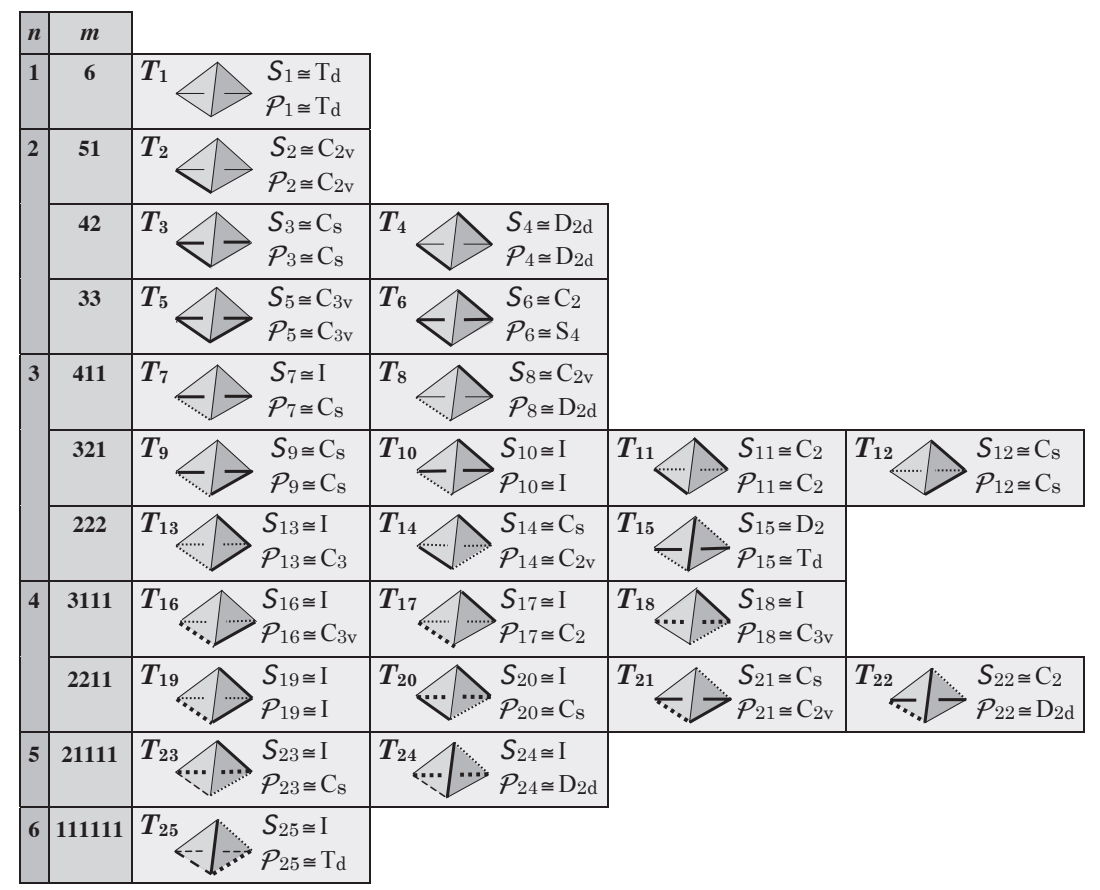

Tab. 1

The expression 'permetry' $p$ is used, because $p$ permutes the edge lengths of a tetrahedron $T$, i.e., the longometry $\lambda(p)$ is an edge lengths permutation. A permetry $p$ where $\lambda(p)$ is the identity, is called a symmetry (again, this uniquely determines a 'symmetry' in its ordinary sense).

Just as the symmetries of a tetrahedron $T$ form a group, so do the permetries and the longometries of $T$. The groups are called symmetry, permetry and longometry group and denoted by $\mathcal{S}, \mathcal{P}$ and $\mathcal{L}$, respectively. The map $\lambda$ which assigns to each permetry $p$ of $T$ the longometry $\lambda(p)$ is a group homomorphism from $\mathcal{P}$ to $\mathcal{L}$ with the kernel $\mathcal{S}$ and, as is well known from group theory, $\mathcal{L}$ and the factor group $\mathcal{P} / \mathcal{S}$ are isomorphic.

We use Schoenfliess symbols (common for symmetry groups in chemistry) to designate both groups $\mathcal{S}$ and $\mathcal{P}$ of a tetrahedron $T$. They are explained in Tab. 2 as subgroups of the full symmetry group of a regular tetrahedron $T_{\text {reg }}$ : Consider a vertex map from $T$ to $T_{\text {reg }}$, where the edges of $T_{\text {reg }}$ have been colored in such a way that there is induced a bijection between equal edge lengths of $T$ and equal edge color of $T_{\text {reg. }}$ The groups $\mathcal{S}$ and $\mathcal{P}$ of $T$ are then isomorphic with the 'color preserving' and with the 'color equality preserving' symmetry group of $T_{\text {reg }}$, respectively.

The Schoenfliess symbols of the symmetry groups $\mathcal{S}_{i}$ and permetry groups $\mathcal{P}_{i}$ of the e-class representatives $T_{i}(1 \leq i \leq 25)$ are shown in the previous Tab. 1. Clearly, all tetrahedrons of $\left[T_{i}\right]_{\mathrm{e}}$ have isomorphic symmetry and isomorphic permetry groups, so that $\mathcal{S}_{i}$ and $\mathcal{P}_{i}$ can be considered to be e-class properties. The elements of this groups are derivable by 


\begin{tabular}{|c|c|c|c|}
\hline two-fo & rotation & mirror reflection & $\begin{array}{l}\text { four-fold rotation } \\
\text { reflection }\end{array}$ \\
\hline \multicolumn{4}{|c|}{ Subgroups of $T_{\text {reg }}$ (group orders in parantheses, $\varepsilon$ denotes the identity element): } \\
\hline (1) & \multicolumn{3}{|c|}{$\varepsilon$ (the group is also named $\mathrm{C}_{1}$ ) } \\
\hline$(2)$ & \multicolumn{3}{|c|}{$\varepsilon$ and 1 two-fold rotation } \\
\hline $\mathbf{C}_{3}$ & \multicolumn{3}{|c|}{$\varepsilon$ and 2 three-fold rotations (around the same axis) } \\
\hline $\mathbf{D}_{2} \quad(4)$ & \multicolumn{3}{|c|}{$\varepsilon$ and 3 two-fold rotations (around different axes) } \\
\hline $\mathbf{C}_{\mathbf{s}} \quad(2)$ & \multicolumn{3}{|c|}{$\varepsilon$ and 1 mirror reflection } \\
\hline $\mathrm{C}_{2 \mathrm{v}} \quad(4)$ & \multicolumn{3}{|c|}{$\begin{array}{l}\varepsilon \text { and } 1 \text { two-fold rotation and } 2 \text { mirror reflections (in planes intersecting at the } \\
\text { rotation axis) }\end{array}$} \\
\hline $\mathbf{C}_{3 \mathrm{v}} \quad(6)$ & \multicolumn{3}{|c|}{$\begin{array}{l}\varepsilon \text { and } 2 \text { three-fold rotations (around the same axis) and } 3 \text { mirror reflections (in } \\
\text { planes intersecting at the rotation axis) }\end{array}$} \\
\hline $\mathbf{S}_{4} \quad(4)$ & \multicolumn{3}{|c|}{$\begin{array}{l}\varepsilon \text { and } 1 \text { two-fold rotation and } 2 \text { four-fold rotation reflections (all around the } \\
\text { same axis) }\end{array}$} \\
\hline $\mathbf{D}_{\mathbf{2 d}} \quad(8)$ & \multicolumn{3}{|c|}{$\begin{array}{l}\varepsilon \text { and } 3 \text { two-fold rotations (around different axes) and } 2 \text { mirror reflections (in } \\
\text { planes intersecting at one of the rotation axes) and } 2 \text { four-fold rotation reflec- } \\
\text { tions (around this axis) }\end{array}$} \\
\hline $\mathbf{T}_{\mathbf{d}}$ & \multicolumn{3}{|c|}{ all 24 symmetries } \\
\hline
\end{tabular}

Tab. 2

'visual coincidence operations' with their representatives $T_{i}$, but can also be generated by a canonizing procedure, which will be summarized here briefly.

The canonizing procedure is based on relational descriptions, or for short descriptions, of the given tetrahedron and an appropriate canonizing algorithm. Since the one we use operates with a minimizing process it will be called minimizing algorithm (see [3], [4], [6]). We explain with an example, namely with the tetrahedron $T$ of the e-class $\left[T_{22}\right]_{\mathrm{e}}$ as shown in Fig. 2 having lengths number $n=4$ and lengths partition $m=2211 ; T$ exists according to Tab. 4 (see Section 4).

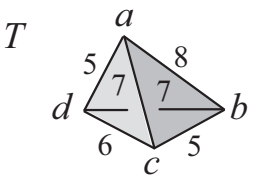

Fig. 2

A first description of $T$, denoted by $\operatorname{Desc}_{1}(T)$, looks as follows:

$\operatorname{Desc}_{1}(T)=(\underbrace{\{a, b, c, d\}}_{=X}, \underbrace{\{a d, d a, b c, c b\}_{5}}_{=R_{1}}, \underbrace{\{c d, d c\}_{6}}_{=R_{2}}, \underbrace{\{a c, c a, b d, d b\}_{7}}_{=R_{3}}, \underbrace{\{a b, b a\}_{8}}_{=R_{4}})$.

This description comprises the vertex set $X$ followed by $n=4$ so-called metric relations $R_{1}, R_{2}, R_{3}$, and $R_{4}$, each of which contains symmetric pairs of vertexes (written without 
brackets and commas) representing equal edge lengths; the metric relations are ordered according to increasing lengths (identified by the indices). The minimizing algorithm now searches for those numberings $\mu: X \rightarrow\{1,2,3,4\}$ which, after lexicographic ordering within each of the numbered $X, R_{1}, R_{2}, R_{3}$, and $R_{4}$, lead to a lexicographically smallest sequence, called the minimal canonization of $T$ and denoted by $\operatorname{Min}(T)$. In this way, $\operatorname{Min}(T)$ results from each of the two numberings $\mu_{1}$ and $\mu_{2}$; they are named minimal numberings:

$$
\begin{gathered}
\operatorname{Min}(T)=\left((1,2,3,4),(12,21,34,43)_{5},(13,31)_{6},(14,23,32,41)_{7},(24,42)_{8}\right)^{1} \\
\text { from } \mu_{1}: a \mapsto 2, b \mapsto 4, c \mapsto 3, d \mapsto 1 \text { or } \mu_{2}: a \mapsto 4, b \mapsto 2, c \mapsto 1, d \mapsto 3 .
\end{gathered}
$$

The two symmetries of $\mathcal{S}_{22} \cong \mathrm{C}_{2}$ are $\mu_{1}^{-1} \mu_{1}$ and $\mu_{2}^{-1} \mu_{1}$. How does one arrive at the eight permetries of $\mathcal{P}_{22} \cong \mathrm{D}_{2 \mathrm{~d}}$ ? The answer is given by the fact that, in addition to $\operatorname{Desc}_{1}(T)=$ ( $X, R_{1}, R_{2}, R_{3}, R_{4}$ ), one can create further descriptions of $T$, all transformable by the minimizing algorithm to the same $\operatorname{Min}(T)$ as already achieved from $\operatorname{Desc}_{1}(T)$. They are obtained by certain permutations of the metric relations $R_{1}, R_{2}, R_{3}$, and $R_{4}$. We find three of them, $\operatorname{Desc}_{2}(T), \operatorname{Desc}_{3}(T)$, and $\operatorname{Desc}_{4}(T)$, each leading to $\operatorname{Min}(T)$ by two minimal numberings (not shown explicitly):

$$
\begin{aligned}
& \operatorname{Desc}_{2}(T)=\left(X, R_{1}, R_{4}, R_{3}, R_{2}\right) \Rightarrow \operatorname{Min}(T) \text { from } \mu_{3} \text { or } \mu_{4}, \\
& \operatorname{Desc}_{3}(T)=\left(X, R_{3}, R_{2}, R_{1}, R_{4}\right) \Rightarrow \operatorname{Min}(T) \text { from } \mu_{5} \text { or } \mu_{6}, \\
& \operatorname{Desc}_{4}(T)=\left(X, R_{3}, R_{4}, R_{1}, R_{2}\right) \Rightarrow \operatorname{Min}(T) \text { from } \mu_{7} \text { or } \mu_{8} .
\end{aligned}
$$

Thus we have eight permetries: the already mentioned $\mu_{1}^{-1} \mu_{1}$ and $\mu_{2}^{-1} \mu_{1}$ (symmetries) together with $\mu_{3}^{-1} \mu_{1}$ and $\mu_{4}^{-1} \mu_{1}$ (coset permetries), $\mu_{5}^{-1} \mu_{1}$ and $\mu_{6}^{-1} \mu_{1}$ (coset permetries), $\mu_{7}^{-1} \mu_{1}$ and $\mu_{8}^{-1} \mu_{1}$ (coset permetries). The involved permutations of the metric relations, namely $\left(R_{1}\right)\left(R_{2}\right)\left(R_{3}\right)\left(R_{4}\right),\left(R_{1}\right)\left(R_{3}\right)\left(R_{2} R_{4}\right),\left(R_{2}\right)\left(R_{4}\right)\left(R_{1} R_{3}\right)$, and $\left(R_{1} R_{3}\right)\left(R_{2} R_{4}\right)$, form a group of order 4 which is isomorphic with the longometry group of $T$.

\section{Remarks.}

(1) It is possible to extend the concepts symmetry, permetry and longometry group to polyhedrons or even to polytopes. These groups may be achieved by the canonizing procedure just illustrated. Such a symmetry group of a $d$-dimensional polytope was in [4] named automorphism group (the elements, being vertex permutations, are the automorphisms of the description). The automorphism group is isomorphic with the 'ordinary symmetry group' (where the elements are the isometries of the whole $d$ dimensional embedding space which map the polytope onto itself). But note that for polytopes, being different from simplexes, the automorphism group alone does not, in general, uniquely determine the kind of the isometries assigned by the isomorphism (for details see [4]).

(2) It should also be mentioned that the canonizing procedure has been applied to generate the symmetry groups of non-rigid figures, which are not easily obtainable by 'visual coincidence operations', but which are of prime importance as models, for instance, of molecular structures (see [1]).

1. It would be sufficient to write only the lexicographically smaller of each symmetric pair. 


\section{Tetrahedron o-classes}

We start again with triangles (the simplexes in 2-space): Isosceles triangles can be subdivided into two classes, the one where the laterals are larger than the base and the one in which they are smaller. By analogy, we consider subdivisions of the e-classes of tetrahedrons:

Definition 3.1. A tetrahedron $T^{\prime}$ belongs to the $o$-class represented by the tetrahedron $T$ and denoted by $[T]_{\mathrm{o}}$ if there exists an e-metry $f$ from $T$ to $T^{\prime}$, so that $\lambda(f)$ preserves the order. We call such an e-metry $f$ an $o$-metry from $T$ to $T^{\prime}$.

Clearly, the o-classes form a partition of all tetrahedrons and make up a refinement of the e-classes. What is the number of o-classes within an e-class $[T]_{\mathrm{e}}$ ?

Theorem 3.1. Let $T$ be a tetrahedron with lengths number $n$ and longometry group $\mathcal{L}$. Then the number $\omega$ of o-classes within the e-class $[T]_{\mathrm{e}}$ is given by $\omega=n ! /|\mathcal{L}|$.

Proof. We use a finite completely tetrahedral set $W$ (see [5]) with $|W|=n$. This assures that all o-classes within $[T]_{\mathrm{e}}$ can be represented by tetrahedrons with edge lengths from $W$ which is achieved as follows: Consider e-metries $f_{k}$ with $1 \leq k \leq n$ ! from a tetrahedron $\mathcal{T}_{1} \in[T]_{\mathrm{e}}$ to tetrahedrons $\mathcal{T}_{k}$ such that (a) all $\mathcal{T}_{k}$ have edge lengths from $W$ and (b) all $\lambda\left(f_{k}\right)$ are mutually different. Clearly, to a fixed $\mathcal{T}_{k}$ and to each e-metry $g$ from $\mathcal{T}_{1}$ to $\mathcal{T}_{k}$ there is assigned a permetry $p$ of $\mathcal{T}_{1}$, such that $p=g^{-1} f_{k}$ and thus (c): $\lambda(p)=\lambda\left(g^{-1} f_{k}\right)=$ $\lambda\left(g^{-1}\right) \lambda\left(f_{k}\right)$. Now, when does $\mathcal{T}_{k}$ belong to the o-class $\left[\mathcal{T}_{1}\right]_{0}$ ? By Definition 3.1 , there must exist an o-metry $g$ from $\mathcal{T}_{1}$ to $\mathcal{T}_{k}$ which, according to (a) and since $\lambda(g)$ preserves the order, is exactly the case if $g$ will be an isometry. But such an isometry $g$ is given if and only if $\lambda\left(g^{-1}\right)$ is the identity which, because of (c), is equivalent to $\lambda\left(f_{k}\right)=\lambda(p)$ or, in other words, to $\lambda\left(f_{k}\right)$ being equal to a longometry of $\mathcal{T}_{1}$. From (b) and since the longometry group $\mathcal{L}$ is an e-class property follows that the o-class $\left[\mathcal{T}_{1}\right]_{\mathrm{o}}$ contains $|\mathcal{L}|$ tetrahedrons $\mathcal{T}_{k}$ and this, of course, is true for each other o-class within $[T]_{\mathrm{e}}$. Hence, $\omega=n ! /|\mathcal{L}|$.

Tab. 3 shows the numbers $\omega_{i}$ of o-classes within the e-classes $\left[T_{i}\right]_{\mathrm{e}}$. By summation over all $\omega_{i}$ one obtains the total number of o-classes, which is 225 .

\begin{tabular}{|c||c|c|c|c|c|c|c|c|c|c|c|c|c|c|c|c|c|c|c|c|c|c|c|c|c|}
\hline$i$ & 1 & 2 & 3 & 4 & 5 & 6 & 7 & 8 & 9 & 10 & 11 & 12 & 13 & 14 & 15 & 16 & 17 & 18 & 19 & 20 & 21 & 22 & 23 & 24 & 25 \\
\hline$\omega_{i}$ & 1 & 2 & 2 & 2 & 2 & 1 & 3 & 3 & 6 & 6 & 6 & 6 & 2 & 3 & 1 & 4 & 12 & 4 & 24 & 12 & 12 & 6 & 60 & 15 & 30 \\
\hline
\end{tabular}

Tab. 3

There are, for instance, $\omega_{22}=6$ o-classes within $\left[T_{22}\right]_{\mathrm{e}}$ represented by the tetrahedrons as shown in Fig. 3, all of which exist according to Tab. 4 (see next Section 4). Note that the 2nd tetrahedron from the right is the tetrahedron $T$ of Fig. 2.
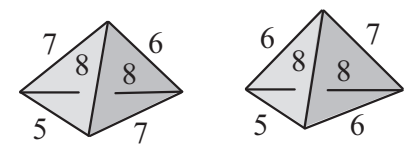
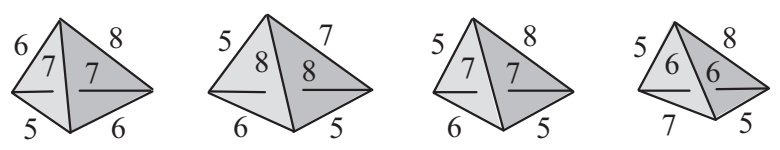

Fig. 3 


\section{Remarks.}

(1) The minimum value is $\omega_{1}=\omega_{6}=\omega_{15}=1$ and, in these cases, the e-classes and o-classes coincide: $\left[T_{1}\right]_{\mathrm{e}}=\left[T_{1}\right]_{\mathrm{o}}$ (regular tetrahedrons), $\left[T_{6}\right]_{\mathrm{e}}=\left[T_{6}\right]_{\mathrm{o}}$ (golden tetrahedrons) and $\left[T_{15}\right]_{\mathrm{e}}=\left[T_{15}\right]_{\mathrm{o}}$ (isosceles tetrahedrons).

(2) The maximum value is $\omega_{23}=60$ which is bigger than $\omega_{25}=30$, even though the tetrahedrons of $\left[T_{25}\right]_{\mathrm{e}}$ have mutually different edge lengths (scalene tetrahedrons).

\section{Smallest p- and q-sets}

In this section we consider o-classes within two simple further classifications which can immediately be recognized by inspecting the first and second column of Tab. 1:

Definition 4.1. A p-class $[T]_{\mathrm{p}}$ or a $q$-class $[T]_{\mathrm{q}}$, where $T$ is a representative, consists of all tetrahedrons with the same lengths partition $m$ as $T$ or the same lengths number (quantity) $n$ as $T$, respectively.

There are 11 p-classes and 6 q-classes, both forming a partition of all tetrahedrons. Of course, the p-classes make up a refinement of the q-classes. Including the e-classes and o-classes we have: $[T]_{\mathrm{q}} \supseteq[T]_{\mathrm{p}} \supseteq[T]_{\mathrm{e}} \supseteq[T]_{\mathrm{o}}$ for any tetrahedron $T$.

First, let us consider the p-class $[T]_{\mathrm{p}}$ where $T$ has lengths partition $m$ and lengths number $n$. We define a smallest $p$-set $P_{m}$ as the set of the $n$ smallest successive integers, such that for each o-class within $[T]_{\mathrm{p}}$ there exists a representative with edge lengths from $P_{m}$. How can $P_{m}$ be determined?

For this purpose we make use of spawning tetrahedrons and digress for a short summary of their features (see also [5]). By definition, a spawning tetrahedron is given according to Fig. 4 with $a \geq b \geq c \geq d \geq e \geq f$.

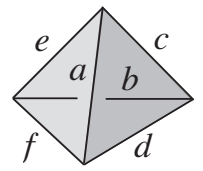

Fig. 4

The attribute 'spawning' is justified by the following remarkable property: All (anisometric) tetrahedrons conceivable by rearranging the edges of a spawning tetrahedron exist. But when do given edge lengths with the order $a \geq b \geq c \geq d \geq e \geq f$ determine a spawning tetrahedron? A necessary and sufficient condition is given by $D>0$ where

$$
D=\left|\begin{array}{ccccc}
0 & a^{2} & e^{2} & c^{2} & 1 \\
a^{2} & 0 & f^{2} & d^{2} & 1 \\
e^{2} & f^{2} & 0 & b^{2} & 1 \\
c^{2} & d^{2} & b^{2} & 0 & 1 \\
1 & 1 & 1 & 1 & 0
\end{array}\right| \quad \text { (Cayley-Menger determinant). }
$$

Of course, the attribute 'spawning', being based on the order of edge lengths, describes an o-class property. There are 32 spawning o-classes since $a \geq b \geq c \geq d \geq e \geq f$ is fulfilled exactly if one of the $2^{5}=32$ possible conditions, resulting from the replacement of ' $\geq$ ' by ' $>$ ' or by ' $=$ ', is fulfilled; in the following we speak of the 32 spawning conditions. Returning to the problem of detecting the smallest p-set $P_{m}$, we explain by means of an example: Let $[T]_{\mathrm{p}}$ be the p-class represented by a tetrahedron $T$ with lengths partition $m=$ 
2211. The determination of $P_{2211}$ is now based on the $4 ! /(2 ! 2 !)=6$ spawning o-classes within $[T]_{\mathrm{p}}$ corresponding to the 6 arrangements of the partition numbers, namely 2211 , $2121,2112,1221,1212$, and 1122 , or to the following 6 spawning conditions, respectively:

$$
\begin{aligned}
& a=b>c=d>e>f, \quad a=b>c>d=e>f, \quad a=b>c>d>e=f, \\
& a>b=c>d=e>f, \quad a>b=c>d>e=f, \quad a>b>c=d>e=f \text {. }
\end{aligned}
$$

In each of these spawning conditions we replace the 4 unequal variables with $u, u+1$, $u+2$, and $u+3$ (while adhering to the order) and form the respective Cayley-Menger determinants, being denoted by $D_{1}, D_{2}, D_{3}, D_{4}, D_{5}$, and $D_{6}$. We then calculate the smallest positive solution $u_{\mathrm{s}}$ of the following system of diophantine inequalities:

$$
D_{1}>0 \wedge D_{2}>0 \wedge D_{3}>0 \wedge D_{4}>0 \wedge D_{5}>0 \wedge D_{6}>0 .
$$

The result $u_{\mathrm{s}}=5$ leads to the smallest p-set $P_{2211}=\{5,6,7,8\}$ as shown in Tab. 4a.

Clearly, each of the $\omega_{19}+\omega_{20}+\omega_{21}+\omega_{22}=54$ o-classes within $[T]_{\mathrm{p}}$ can be represented by exactly one tetrahedron with edge lengths from $P_{2211}$. The 54 tetrahedrons include all anisometric tetrahedrons of $[T]_{\mathrm{p}}$ conceivable with edge lengths from $P_{2211}$. Among these 54 tetrahedrons, 6 are spawning each generating 9 tetrahedrons by rearranging the edges. In general: Within a p-class, the number of spawning o-classes is a divisor of the number of

\begin{tabular}{|c|c|c|c|c|c|}
\hline \multicolumn{3}{|c|}{ Tab. $4 \mathrm{a}$} & \multicolumn{3}{|c|}{ Tab. 4b } \\
\hline $\begin{array}{c}\text { lengths } \\
\text { partition } m\end{array}$ & smallest p-set $P_{m}$ & $\begin{array}{c}\text { number of } \\
\text { o-classes: } \\
\text { all (spawning) }\end{array}$ & $\begin{array}{l}\text { lengths } \\
\text { number } n\end{array}$ & smallest q-set $Q_{n}$ & $\begin{array}{c}\text { number of } \\
\text { o-classes: } \\
\text { all (spawning) }\end{array}$ \\
\hline 6 & $\{1\}$ & 1 (1) & 1 & $\{1\}$ & $1(1)$ \\
\hline 51 & $\{2,3\}$ & $2(2)$ & \multirow{3}{*}{2} & \multirow{3}{*}{3,4} & \multirow{3}{*}{$9(5)$} \\
\hline 42 & $\{3,4\}$ & $4(2)$ & & & \\
\hline 33 & $\{2,3\}$ & $3(1)$ & & & \\
\hline 411 & $\{4,5,6\}$ & $6 \quad(3)$ & \multirow{3}{*}{3} & \multirow{3}{*}{$5,6,7$} & \multirow{3}{*}{$36(10)$} \\
\hline 321 & $\{5,6,7\}$ & $24 \quad(6)$ & & & \\
\hline 222 & $\{4,5,6\}$ & $6(1)$ & & & \\
\hline 3111 & $\{6,7,8,9\}$ & $20 \quad(4)$ & \multirow{2}{*}{4} & \multirow{2}{*}{$6,7,8,9$} & \multirow{2}{*}{$74(10)$} \\
\hline 2211 & $\{5,6,7,8\}$ & $54(6)$ & & & \\
\hline 21111 & $\{7,8,9,10,11\}$ & $75 \quad(5)$ & 5 & $\{7,8,9,10,11\}$ & $75 \quad(5)$ \\
\hline 111111 & $\{7,8,9,10,11,12\}$ & $30(1)$ & 6 & $\{7,8,9,10,11,12\}$ & $30 \quad(1)$ \\
\hline \multicolumn{2}{|c|}{ total of o-classes: all (spawning) } & $225(32)$ & \multicolumn{2}{|c|}{ total of o-classes: all (spawning) } & $225(32)$ \\
\hline
\end{tabular}
o-classes because the quotient stands for the number of possible anisometric tetrahedrons with given edge lengths (see third column of Tab. 4a and also [5]).

Tab. 4

We now turn to Tab. 4b. Let $[T]_{\mathrm{q}}$ be the q-class where $T$ has lengths number $n$. By analogy to a smallest p-set $P_{m}$, we define a smallest $q$-set $Q_{n}$ as the set of the $n$ smallest successive integers such that for each o-class within $[T]_{\mathrm{q}}$ there exists a representative with edge lengths from $Q_{n}$. There are $\left(\begin{array}{c}5 \\ n-1\end{array}\right)$ spawning o-classes within $[T]_{\mathrm{q}}$ corresponding to the spawning conditions with $n-1$ signs ' $>$ ' and $6-n$ signs ' $=$ '. A smallest q-set $Q_{n}$ in Tab. $4 \mathrm{~b}$ is obtained by determining the smallest positive solution of the system of the $\left(\begin{array}{c}5 \\ n-1\end{array}\right)$ 
respective diophantine inequalities. The number of o-classes within $[T]_{\mathrm{q}}$ results from summation over the appropriate $\omega_{i}$. For $n=4$, for instance, we have 74 o-classes and each of them can be represented by exactly one tetrahedron with the property that only the 4 edge lengths of $Q_{4}$ occur. And again, these 74 tetrahedrons make up all conceivable anisometric tetrahedrons with this property.

Remark. The reader may see that the calculation of the smallest p- and q-sets could be simplified should the following conjecture be true: If in a tetrahedron all 6 edges are extended by the same length $t$ (in our case by $t=1$ ), then the resulting edges, being arranged in the same way, again determine a tetrahedron.

\section{Higher dimensions}

A generalization in $d$-dimensional spaces may be considered. We counted the numbers of the simplex q-, p-, e- and o-classes denoted by $\mathrm{q}(d), \mathrm{p}(d), \mathrm{e}(d)$, and $\mathrm{o}(d)$, respectively. These numbers can be calculated as follows: $\mathrm{q}(d)=\left(\begin{array}{c}d+1 \\ 2\end{array}\right)$ and $\mathrm{p}(d)$ (partitions of a natural number) is obtained from a generating function well-known in combinatorics; for the determination of $\mathrm{e}(d)$ we used DeBruijn's generalization of Polya's theory of counting and for $\mathrm{o}(d)$ Polya's theory itself. In Tab. 5 these numbers are given for $1 \leq d \leq 7$.

\begin{tabular}{|l|l|l|l|l|}
\hline$d$ & $\mathrm{q}(d)$ & $\mathrm{p}(d)$ & $\mathrm{e}(d)$ & \multicolumn{1}{c|}{$\mathrm{o}(d)$} \\
\hline 1 & 1 & 1 & 1 & 1 \\
\hline 2 & 3 & 3 & 3 & 4 \\
\hline 3 & 6 & 11 & 25 & 225 \\
\hline 4 & 10 & 42 & $1 ' 299$ & $856^{\prime} 608$ \\
\hline 5 & 15 & 176 & $11^{\prime} 974^{\prime} 452$ & $319^{\prime} 872^{\prime} 163^{\prime} 585$ \\
\hline 6 & 21 & 792 & $94^{\prime} 345^{\prime} 468^{\prime} 975$ & $16^{\prime} 096^{\prime} 217^{\prime} 596^{\prime} 356^{\prime} 372^{\prime} 660$ \\
\hline 7 & 28 & 3718 & $152^{\prime} 799^{\prime} 292^{\prime} 695^{\prime} 935^{\prime} 115$ & $156^{\prime} 189^{\prime} 537^{\prime} 129^{\prime} 127^{\prime} 582^{\prime} 748^{\prime} 089^{\prime} 210^{\prime} 443$ \\
\hline
\end{tabular}

Tab. 5

\section{References}

[1] Floersheim, P.; Wirth, K.; Huber, M.K.; Pazis, D.; Siegerist, F.; Haegi, H.R.; Dreiding, A.S.: From Mobile Molecules to their Symmetry Groups: A Computer-Implemented Method. Stud. Phys. Theor. Chem. 23 (1983), 59-80.

[2] Rassat, K.; Fowler, P.W.: Is There a "Most Chiral Tetrahedron"? Chem. Eur. J. 10 (2004), 6575-6580.

[3] Wirth, K.: Coding of Relational Descriptions of Molecular Structures. J. Chem. Inf. Comput. Sci. 26 (1986), 242-249.

[4] Wirth, K.; Dreiding, A.S.: Kants Hand, Chiralität und konvexe Polytope. Elem. Math. 62 (2007), 8-29. English version under http: / /dx.doi.org/10.5167/uzh-59264

[5] Wirth, K.; Dreiding, A.S.: Edge lengths determining tetrahedrons. Elem. Math. 64 (2009), 160-170.

[6] Wirth, K.; Huber, M.K.: Numbering of Finite Relational Systems. Match 12 (1981), 3-14.

Karl Wirth, André S. Dreiding

Organisch-Chemisches Institut, Universität Zürich

Winterthurerstrasse 190, CH-8057 Zürich, Switzerland

e-mail: wirthk@gmx.ch, asd@oci.uzh.ch 